\section{Obeticholic acid}

Aust Prescr 2020;43:174-5 https://doi.org/10.18773/ austprescr.2020.063

\section{$<$}

Some of the views expressed on newly approved products should be regarded as preliminary, as there may be limited published data at the time of publication, and little experience in Australia of their safety or efficacy. However, the Editorial Executive Committee believes that comments made in good faith at an early stage may still be of value. Before new drugs are prescribed, the Committee believes it is important that more detailed information is obtained from the manufacturer's approved product information, a drug information centre or some other appropriate source.

\section{Approved indication: primary biliary cholangitis Ocaliva (Chiesi) $5 \mathrm{mg}$ and $10 \mathrm{mg}$ tablets}

Primary biliary cholangitis is an autoimmune condition in which the bile ductules in the liver are destroyed. Cholestasis and inflammation cause altered liver function with raised concentrations of alkaline phosphatase. The damage leads to cirrhosis, hence the condition is also known as primary biliary cirrhosis. Treatment with ursodeoxycholic acid can slow progression and delay the need for a liver transplant.

The regulation of bile acids involves the farnesoid $X$ nuclear receptor. Activating the receptor reduces the concentrations of bile acids in the liver. This leads to reduced inflammation and fibrosis. Obeticholic acid acts as an agonist on this receptor.

After absorption obeticholic acid is conjugated with glycine or taurine and secreted into bile. The conjugates can be reabsorbed from the gut, setting up an enterohepatic recirculation. Most of the dose is eventually excreted in the faeces. If the patient has moderate or severe hepatic impairment (Child-Pugh Class B and C), the usual starting dose of $5 \mathrm{mg}$ daily is reduced to $5 \mathrm{mg}$ weekly. In patients taking warfarin, obeticholic acid will decrease the INR. Bile acid binding resins should not be taken within 4-6 hours of obeticholic acid.

Daily doses of $10 \mathrm{mg}, 25 \mathrm{mg}, 50 \mathrm{mg}$ or placebo were studied in a phase II trial involving 165 patients with primary biliary cirrhosis that was not well controlled by ursodeoxycholic acid. These patients were treated for three months. The mean concentrations of alkaline phosphatase were reduced by $21-25 \%$ with obeticholic acid but only by $3 \%$ with placebo. This biochemical benefit was maintained in the 78 patients who continued treatment in a 12-month extension of the trial. ${ }^{1}$ While the higher doses had similar efficacy to the $10 \mathrm{mg}$ dose, they were more frequently associated with severe pruritus so they were not used in a phase III trial.

The double-blind phase III trial randomised 73 patients to take obeticholic acid $10 \mathrm{mg}$ daily and 70 patients to take $5 \mathrm{mg}$ for six months then increase to $10 \mathrm{mg}$ according to the response and adverse effects. Another 73 patients took a placebo. If tolerated, all patients continued to take ursodeoxycholic acid. The primary end point of the study was at least a $15 \%$ reduction in alkaline phosphatase, with a concentration less than 1.67 times the upper limit of normal, and a total bilirubin concentration no higher than the upper limit of normal. After one year this composite end point had been achieved by $47 \%$ of the $10 \mathrm{mg}$ group and $46 \%$ of the $5-10 \mathrm{mg}$ group. This was statistically better than the $10 \%$ response in the placebo group. ${ }^{2}$ Following the double-blind phase, 193 patients entered an extension study. They were given openlabel obeticholic acid $5 \mathrm{mg}$ which could be increased after three months according to the response. The proportions of patients achieving the primary end point were $53 \%$ at 24 months, $55 \%$ at 36 months and 51\% at 48 months. Concentrations of alkaline phosphatase and other liver enzymes were significantly reduced. ${ }^{3}$

In the long-term extension study the most frequent adverse effect was pruritis affecting $77 \%$ of the patients. Other common adverse events included fatigue, urinary tract infection, headache and arthralgia. ${ }^{3}$ There is a risk of liver-related adverse reactions. These include flare-ups of cholangitis, jaundice and ascites. The risk of these adverse events increases with the dose of obeticholic acid so $10 \mathrm{mg}$ daily is the highest recommended dose. Possibly because of its action on bile acids, obeticholic acid has an effect on lipids. Concentrations of low-density lipoprotein may increase and high-density lipoprotein cholesterol may decrease. ${ }^{2}$

If primary biliary cholangitis does not respond adequately to ursodeoxycholic acid, or if the patient cannot tolerate it, obeticholic acid can be added to therapy. Most patients will then have a reduction in alkaline phosphatase concentrations, but the clinical consequences are less clear. In the double-blind phase III trial there was no difference in liver fibrosis between obeticholic acid and placebo. ${ }^{2}$ The 10 -year predicted risk of death or liver transplantation only reduced slightly, from $20 \%$ at baseline to $18.95 \%$ at 48 months. ${ }^{3}$ As the patients in the trial had relatively early disease, it is uncertain what effect obeticholic acid will have in more advanced disease. It will take several years before it is known whether or not obeticholic acid has any clinical benefit in primary biliary cholangitis.

\section{$\mathbf{T}$ manufacturer provided the product information}

\section{REFERENCES}

1. Hirschfield GM, Mason A, Luketic V, Lindor K, Gordon SC, Mayo M, et al. Efficacy of obeticholic acid in patients with primary biliary cirrhosis and inadequate response to ursodeoxycholic acid. Gastroenterology 2015;148:751-61.e8. https://doi.org/10.1053/j.gastro.2014.12.005

2. Nevens F, Andreone P, Mazzella G, Strasser SI, Bowlus C, Invernizzi P, et al; POISE Study Group. A placebo-controlled trial of obeticholic acid in primary biliary cholangitis. N Engl J Med 2016;375:631-43. https://doi.org/10.1056/ NEJMoa1509840

3. Trauner M, Nevens F, Shiffman ML, Drenth JPH, Bowlus CL Vargas $V$, et al. Long-term efficacy and safety of obeticholic acid for patients with primary biliary cholangitis: 3-year results of an international open-label extension study. Lancet Gastroenterol Hepatol 2019;4:445-53. https://doi.org/ $10.1016 / s 2468-1253(19) 30094-9$ 
<ustralian Prescriber

VOLUME 43 : NUMBER 5 : OCTOBER 2020

The Transparency Score is explained in New drugs: transparency, Vol 37 No 1, Aust Prescr 2014;37:27.

At the time the comment was prepared, information about this drug was available on the websites of the Food and Drug Administration in the USA, and the European Medicines Agency. 